\title{
La rigueur et l'innovation d'Harold E. Palmer (1877-1949) : Le cas des exercices dans ses propositions didactiques
}

The rigour and innovation of Harold E. Palmer (1877-1949): the case of exercises in his didactic proposals

\section{Georges Daniel Véronique}

\section{(2) OpenEdition \\ Journals}

\section{Édition électronique}

URL : https://journals.openedition.org/dhfles/6846

DOI : $10.4000 /$ dhfles.6846

ISSN : 2221-4038

\section{Éditeur}

Société Internationale pour l'Histoire du Français Langue Étrangère ou Seconde

\section{Édition imprimée}

Date de publication : 1 décembre 2019

Pagination : 429-448

ISBN : 0992-7654

ISSN : 0992-7654

\section{Référence électronique}

Georges Daniel Véronique, «La rigueur et l'innovation d'Harold E. Palmer (1877-1949) : Le cas des exercices dans ses propositions didactiques ", Documents pour l'histoire du français langue étrangère ou seconde [En ligne], 62-63 | 2019, mis en ligne le 12 avril 2020, consulté le 27 mars 2023. URL : http:// journals.openedition.org/dhfles/6846; DOI : https://doi.org/10.4000/dhfles.6846 


\title{
La rigueur et l'innovation d'Harold E. Palmer (1877-1949) : Le cas des exercices dans ses propositions didactiques
}

\author{
The rigour and innovation of Harold E. Palmer (1877-1949): the case of exercises \\ in his didactic proposals
}

Georges Daniel Véronique

\section{Introduction}

1 L'œuvre didactique de Harold E. Palmer (1877-1949) s'inscrit de façon singulière dans le grand mouvement de réforme qui traverse l'enseignement des langues modernes en Europe et aux États-Unis à partir des années 1870. En effet, Palmer, qui est manifestement un continuateur des pionniers qui ont initié le mouvement, poursuit également un projet original d'enseignement des langues étrangères, ce qu'il nomme à ses débuts, la méthode ${ }^{1}$ Palmer (Smith 2004 : 98). Grâce à la remarquable thèse de Richard Smith (2004) et à ses publications (Smith 1999, 2011), nous disposons dorénavant d'une bonne information sur la vie et l'œuvre de Palmer². Je puiserai abondamment dans ses travaux, qui éclairent l'itinéraire de l'enseignant d'exception qu'était Palmer. Je partage l'idée de Smith (2011) que Palmer défend une vision originale de l'enseignement des langues étrangères, nullement réductible à la pratique postérieure de l'applied linguistics américaine. Sa démarche est certainement plus proche de la didactique des langues étrangères, telle qu'elle sera définie dans les travaux de langue française, à la fin du XXe siècle (Véronique 2009); cependant, je me garderai également de cet autre anachronisme.

2 Après avoir situé Palmer et son œuvre au sein du mouvement de réforme de l'enseignement des langues (1880 - 1920), j'exposerai, à la suite de Véronique (1983, 
1992), et de Smith $(2004,2011)$, les thèses linguistiques et les principes pédagogiques qui organisent la démarche de Palmer. J'aborderai ensuite, ses propositions en matière d'exercices pour l'enseignement. Je conclurai cet article, par une évaluation de l'apport de Palmer au développement d'un enseignement-apprentissage scientifique des langues au XXe siècle.

Ce texte se fondera sur l'examen des ouvrages suivants de Palmer: The scientific study and teaching of languages (1917) (abrégé en SSTL), The principles of language study (1921a) (abrégé en PLS), The oral method of teaching languages (abrégé en OM) (1921b), Memorandum on problems of English teaching in the light of a new theory (1924) (désormais Memorandum), This Language learning business (abrégé en LLB), publié en 1932 avec V. Redman, et The Teaching of Oral English (abrégé en TOE) (1940). Les préoccupations pédagogiques de Palmer et sa réflexion sur les exercices ont fortement contribué à l'établissement d'un enseignement renouvelé des langues vivantes. L'interrogation sur l'inscription de Palmer dans l'histoire de la didactique des langues constituera le fil conducteur de cette contribution.

\section{Palmer et le mouvement de réforme de l'enseignement des langues $(1880-1920)$}

Smith $(2004,2011)$ relève à juste titre que l'œuvre de Palmer doit être périodisée en fonction des lieux où il a exercé - en Belgique (1902-1914), à Londres (1915-21, puis de 1936 à la fin de sa vie) et au Japon (1922-1936) - et des dominantes successives de ses travaux - la phase des innovations pédagogiques: de 1900 aux années 20, la phase des recherches à l'étranger: avant 1914 et dans les années 20 et 30, et la phase de recentrage au Royaume-Uni : les années 30 et 40 . Dans ce qui suit, je m'intéresserai à la période des innovations pédagogiques et des propositions que formule Palmer sur l'enseignement et l'apprentissage des langues étrangères - cela correspond principalement aux travaux menés entre 1915 et 1925. Je négligerai de ce fait, ses travaux de lexicologie, qui arrivent plus tard dans son œuvre.

\section{Des éléments biographiques et bibliographiques}

5 Palmer se distingue de ses prédécesseurs par sa formation initiale, son engagement professionnel et la diversité de ses intérêts en matière de «science de la pédagogie linguistique ». S'il est polyglotte, il n'a aucune formation en grammaire comparée ; il n'est pas néo-grammairien, contrairement à Henry Sweet (1845-1912) ou Wilhelm Viëtor (1850-1918). C'est un phonéticien autodidacte qui adhère à l'Association Phonétique Internationale dès $1907^{3}$, et qui se forme à la linguistique, principalement à travers la lecture du Cours de Ferdinand de Saussure (1857 - 1913). Il entretient des relations avec Albert Sechehaye (1870-1946), Edward Sapir (1884-1934) et Leonard Bloomfield (1887-1949). Sa vie durant, Palmer étudie des langues non européennes. Palmer maîtrisait le français, appris très tôt à l'école, et l'espéranto ; dans ses ouvrages, il cite des exemples en anglais, en allemand, en néerlandais, en français et en espagnol Selon le phonéticien Daniel Jones (1881-1967), cité par Smith (2004 : 105), Palmer est un " accomplished French scholar and a fine language teacher ${ }^{4}$ "; il ajoute qu'il possède un " outstanding talent for linguistic theory and pedagogy ${ }^{5}$ ». 
6 Palmer est très engagé dans l'enseignement de l'anglais et du français, langues étrangères, à l'étranger. Entre 1906 et 1952 - date de parution du dernier cours posthume - il publie de nombreux manuels d'anglais, de français et d'espéranto. Palmer a une pratique d'angliciste éprouvé - il donne en 1924, a Grammar of spoken English, en 1926, en collaboration, Dictionary of English pronunciation with American variants et en 1938, a Grammar of English words, travail lexicographique original notamment par le dégroupement des unités en fonction de critères sémantiques et syntaxiques, et par le choix des énoncés-exemples qui indiquent les compatibilités du mot. Il est également l'auteur de Principles of Romanization pour le japonais en 1930.

7 Quand Palmer débute dans l'enseignement des langues étrangère, le mouvement de réforme est déjà en place. À ses débuts, il s'inspire de la méthode d'enseignement de Maximilian Delphinus Berlitz (1852-1921) et de celle de Thomas Prendergast (1806-1886), pour élaborer sa propre démarche pédagogique. Palmer est plus jeune que les initiateurs du mouvement de réforme de l'enseignement des langues. Il appartient à la génération qui succède à celle de Sweet, de Viëtor, d'Otto Jespersen (1860-1943) et de Paul Passy (1859-1940) ; Palmer lit assidûment tous ces auteurs.

\section{Palmer et la méthodologie directe}

Palmer (1922) considère que la méthodologie directe devait :

- développer l'étude systématique et rationnelle de la prononciation à l'aide de la science phonétique,

- propager l'idée que la langue est d'abord le véhicule des idées,

- et défendre la thèse que l'enseignement des langues étrangères doit utiliser des méthodes simulant l'apprentissage de la langue maternelle, mais qu'elle n'a atteint ses objectifs que partiellement. Selon lui, les partisans de la méthode directe se sont trompés de cible. Ils ont rejeté la traduction et l'emploi de la langue maternelle dans la classe de langue alors qu'il fallait dénoncer "(...) l'attention excessive de l'ancienne méthode à la construction grammaticale » (PLS : 124-126).

Palmer s'oppose à ses prédécesseurs sur quelques points. Il rejette le dogmatisme de Sweet qui survalorise la place de la phonétique dans l'enseignement des langues et se sépare de Berlitz sur le recours à la traduction ( $L L B: 53$ ). Il prend également ses distances par rapport à la méthode naturelle de François Gouin (1831-1896), bien qu'il considère qu'un apprenant de langue étrangère doive être encouragé à récupérer ses capacités spontanées d'apprentissage des langues (PLS, chapitre 1). En fonction du principe de concrétude (concreteness) (cf. plus loin), il se propose de réhabiliter l'emploi de la langue maternelle et de la traduction dans l'enseignement. Dans OM (1921: 6-7), Palmer renvoie dos à dos les tenants d'une méthode directe stricte et les partisans irréductibles de la priorité à l'oral (cf. infra Tabl.). Plus tard, il rejettera également le recours au British American Commercial Industrial Scientific (BASIC) English de Ogden \& Richards ainsi que la méthode de Michael West qui privilégie la lecture comme moyen d'apprentissage des langues étrangères (LLB : pp. 67-73).

(Tabl.) : La méthodologie directe et la méthodologie orale d'après Palmer (OM : 6-7)

\begin{tabular}{|l|l|}
\hline La méthode directe & La méthode orale \\
\hline
\end{tabular}




\begin{tabular}{|l|l|}
\hline $\begin{array}{l}\text { Exclut le recours à l'orthographe usuelle dans un } \\
\text { premier temps. }\end{array}$ & Exclut le recours à tout système scriptural. \\
\hline $\begin{array}{l}\text { Emploie des textes suivis (dialogues, descriptions, } \\
\text { narrations) élaborés selon des critères de facilité, } \\
\text { de naturel et d'intérêt. }\end{array}$ & $\begin{array}{l}\text { N'emploie aucun matériau de lecture (reading } \\
\text { matter). }\end{array}$ \\
\hline $\begin{array}{l}\text { Définit un programme explicite et conscient } \\
\text { d'enseignement. }\end{array}$ & $\begin{array}{l}\text { Emploie des techniques qui entraînent et } \\
\text { développent les capacités d'assimilation } \\
\text { inconsciente des élèves. }\end{array}$ \\
\hline $\begin{array}{l}\text { Cultive les capacités d'analyse et de synthèse des } \\
\text { élèves. }\end{array}$ & $\begin{array}{l}\text { Développe des facultés de mémorisation et } \\
\text { d'assimilation des nouvelles habitudes } \\
\text { linguistiques (habit-forming). }\end{array}$ \\
\hline Met en place un travail audio-oral et visuel. & \begin{tabular}{l} 
Instaure un travail principalement audio-oral. \\
\hline
\end{tabular} \\
\hline
\end{tabular}

10 Le cheminement de Palmer dans l'élaboration d'une pédagogie nouvelle des langues étrangères est singulier et original. D'une part, il affirme son ambition d'ériger la pédagogie des langues en science et, d'autre part, il formule des propositions pédagogiques très précises, qui semblent avoir été éprouvées dans des lieux d'enseignement.

\section{La pédagogie scientifique des langues de Palmer}

11 Dans la plupart de ses ouvrages, Palmer insiste sur « [...] la nécessité d'une codification rationnelle de la pédagogie linguistique » (LLB: 10) et sur le fait que «[...] l'art de rédiger des manuels est encore dans l'enfance » (PLS:34). Sa démarche méthodologique explore les deux versants de l'apprentissage (learning) et de l'enseignement (teaching) des langues. Palmer adhère à l'idée d'une organisation rationnelle de l'enseignement des langues prônée par la méthode directe. Il propose des procédures pour organiser la sélection, la gradation et la progression des contenus linguistiques à enseigner. Sa démarche pédagogique est nourrie de recherches phonétiques, de la linguistique émergente et d'une psychologie de l'apprentissage associationniste.

\section{Une conception du langage et de la langue à enseigner}

Dans la terminologie de Palmer, speech (dans le contexte d'art of speech) désigne la faculté de langage et apparaît comme un équivalent de "langage ». Language code ou language correspond à la " langue » et «[...] that aspect of language which is not code » (cet aspect de la langue qui n'est pas le code), à la " parole » saussurienne. Il distingue deux niveaux dans les fonctionnements langagiers : i) un basic speech material (un matériau linguistique de base) ou primary matter (matériau premier) - les unités qui se prêtent à la composition et à la dérivation, (les pliologs dans la terminologie de Palmer) et des constructions - et ii) un secondary matter (un matériau second), nommé également secondary speech (langage second), qui comprend la dimension écrite de la langue. 
Voici le commentaire éloquent qu'adresse Sechehaye à Palmer, à ce propos, dans une lettre écrite en juillet 1933, à l'occasion de l'anniversaire de ce dernier :

Dans votre carrière déjà si bien remplie vous avez constamment cherché à associer étroitement les doctrines de la méthodologie à l'analyse des faits de langage. Vous avez esquissé ou élaboré beaucoup de théories. Je vois plusieurs points sur lesquels vous avez réussi à marquer d'une façon frappante cette interdépendance étroite de la science et de l'enseignement. J'en signalerai ici trois.

Il y a d'abord la fameuse distinction saussurienne entre la langue et la parole. [...] Vous l'avez saisie par l'angle qui vous intéressait et vous opposez l'analyse intellectuelle du système de la langue le manuel de grammaire descriptive avec ses règles, ses tableaux et ses définitions, à l'usage vivant d'un langage organisé fondé sur un ensemble de réflexes solidement imprimés dans notre matière cérébrale. Cet usage vivant étant ce qu'il est, vous avez osé avoir recours aux procédés du « drill », procédés entièrement opposés aux habitudes intellectualisantes de l'école. [...]

Cependant ce «drill » doit être ordonné ; il s'agit de l'organiser de la façon la plus économique et la plus profitable. Dans votre premier ouvrage, The Scientific Study of Languages, vous aviez déjà esquissé un tableau des « ergoniques » du français, et de ses types de structures. Depuis lors ce problème du mécanisme grammatical, de ses éléments structurels et des rapports qu'ils entretiennent naturellement entre eux n'a jamais cessé de vous préoccuper. (1934:11)

On apprécie la perspicacité du commentaire de Sechehaye qui comprend toute l'originalité de la démarche de Palmer, ce méthodologue qui contribue à « la précieuses collaboration de la science pure et de la pratique » $(1934: 14)$.

En matière grammaticale et lexicale, Palmer développe une analyse originale en constituants immédiats. Il nomme ergonics ou mechanism grammar, une discipline qui a pour objet de «classer les unités de la langue selon leur fonction dans la phrase » et " construire des unités originales inconnues à partir des plus petites unités qui les composent » (SSTL : 226). Cette démarche pourrait lui avoir été inspirée par la notion de substitution, empruntée à Prendergast (Smith 2004 : 116). Cette démarche innovante précède largement les travaux de la linguistique distributionnaliste américaine des années quarante, qui développera une démarche analogue (Fries 1952).

L'ergonics allie " analyse et synthèse, de la phrase aux unités minimales non sécables » (SSTL : 226). Palmer crée une terminologie originale pour les différents types d'unités dégagés, de la marque intonative ou alog(ism) à l'affixe et au mot fonctionnel miolog, au monolog (le mot en une seule unité), au polylog (plusieurs monologs associés) et au schème syntaxique ou construction pattern. Il emploie collocation, pour les syntagmes, peu ou prou, en voie de figement et les lexies ( $L L B$ :128-131). En matière lexicale, il étudie les mots simples ou monologs, les composés et les dérivés ou pliologs et les locutions.

17 Palmer pense l'ergonics comme une machinerie permettant de "générer » les phrases d'une langue par la spécification de la combinatoire des unités identifiées (de l'alog[ism] à la construction), à la manière des guides Chaix ou Bradshaw pour les chemins de fer. Il existe deux composantes dans les tables ergoniques: l'inventaire (catalogue) et le tableau des combinatoires (Chart). 


\section{Une conception de l'apprentissage linguistique}

Palmer postule une compétence innée d'acquisition des langues. Dans PLS et dans LLB, il expose ainsi sa conception de l'appropriation des langues :

- l'homme dispose d'une capacité (angl. capacity) naturelle et innée à user des langues à l'oral.

- Il dispose de facultés spontanées d'assimilation (angl. spontaneous capacities of assimilation) qui doivent être stimulées lors de tout nouvel apprentissage linguistique (PLS :127).

Selon Palmer, «l'essence de l'apprentissage d'une langue, c'est la formation de liens entre les symboles constituant une langue et les concepts, les notions ou les idées qu'ils représentent (stand for) (LLB: 97). Cette phase de fusion, précédée d'une phase d'identification, se réalise à l'aide de l'apprentissage par cœur. Palmer rappelle que lors de l'acquisition du langage (L1), l'enfant acquiert la memorized matter (la matière mémorisée) ou le primary speech (langage premier), grâce à des primary speech habits (des habitudes linguistiques premières) comme l'oral observation (l'observation orale), l'oral mimicry (l'imitation orale), ou l'oral mechanizing (l'automatisation orale), qu'il s'agit de réactiver lors de l'appropriation d'une langue étrangère. Pour Palmer, il faut conjoindre un apprentissage implicite du matériau premier (primary matter) de la langue cible et un enseignement explicite, en sollicitant les capacités d'analyse et de synthèse des élèves, notamment grâce à l'écrit.

20 À l'instar des habitudes (primary speech habits) identifiées chez l'enfant, Palmer postule des capacités identiques chez l'apprenant d'une langue étrangère qu'il s'agit de développer (Memorandum : 13 et suivantes). Il se propose ainsi d'intervenir aussi bien sur les habitudes primaires à transférer en langue étrangère que sur les habitudes d'observation visuelle, d'imitation graphique ou de mécanisation graphique des élèves. Dans le Memorandum, Palmer identifie six habitudes associées au traitement de l'oral, dont par exemple, la capacité de dérivation (Memorandum : 20), et six habitudes liées à l'apprentissage de l'écrit, dont par exemple l'observation visuelle (Memorandum : 34), qu'il s'agit d'entraîner.

21 Palmer est proche de l'associationnisme de Wilhelm Wundt (1832-1920) et de Sechehaye, tel que ce dernier l'a exprimé dans Programme et méthodes de la linguistique théorique - Psychologie du langage(1908). Il partage les critiques de ce dernier à l'égard de Wundt sur sa non prise en compte du fait grammatical.

\section{Quelques principes généraux pour une pédagogie linguistique scientifique}

Dans SSTL, et dans d'autres ouvrages, Palmer formule les principes généraux d'une pédagogie linguistique fondée sur la caténisation ou apprentissage par cœur, la sémantisation et la ségrégation. L'accès au sens se fait selon des procédés de monstration, de traduction, de paraphrase et de contextualisation. Palmer prône une gradation stricte et une progression rationnelle.

La définition d'un programme d'enseignement linguistique doit reposer sur une prise en compte de l'apprenant, de la nature de l'enseignement à dispenser et de principes 
généraux, dont je rappelle quelques éléments ci-dessous. Véronique $(1983,1992)$ et Smith $(2004,2011)$ les commentent plus longuement.

i. Dans les débuts, l'enseignement est organisé de telle sorte que l'étudiant recouvre ses facultés spontanées d'acquisition du langage. On l'entraîne tout d'abord à affiner ses capacités de réception et de rétention des messages oraux, puis à articuler des séquences de sons en langue seconde, et enfin, à développer ses capacités mimiques. Il s'agit de mobiliser ses capacités de mémorisation mais aussi celles d'assimilation inconsciente (PLS, ch. 7, SSTL: 91).

ii. Dans l'étude consciente initiale de la langue (on dirait explicite de façon contemporaine), il convient d'appliquer un principe de ségrégation (SSTL: 72), c'est-à-dire de séparation des dimensions phonétique, sémantique et ergonique ; ce qu'il est impossible d'accomplir dans une démarche d'étude inconsciente (on dirait implicite de nos jours) ou à des stades ultérieurs de l'étude consciente (ou explicite). Ce principe est décrit sous le terme de «multiple line of approach» (une approche multilinéaire) dans le Memorandum et dans PLS (108 et suivantes).

iii. Le but de l'enseignement est de faire de telle sorte que l'étudiant associe étroitement (angl. fuse) les éléments de l'expérience et leurs équivalents linguistiques dès le premier cours (PLS : 103). On s'appliquera par un travail intensif de mémorisation à créer de nouvelles habitudes langagières ou à réutiliser des habitudes antérieurement acquises (PLS, ch. 8). Il faudra enseigner à produire des énoncés appropriés aux situations sans avoir recours à des explications métalinguistiques.

iv. La caténisation ou apprentissage par cœur est la base de tout apprentissage des langues. Quand le noyau de la langue est appris par cœur, la maîtrise croît selon un processus de boule de neige. Ce type d'apprentissage prévient l'erreur, évite l'analyse et rend la matière linguistique immédiatement disponible (SSTL, section 15).

v. L'accès au sens ou sémantisation se fera selon les procédés suivants: la monstration, la traduction, la paraphrase et la contextualisation (SSTL : 49 et sq., PLS : 12). La sémantisation n'est qu'un des aspects du principe de concrétude (concreteness), qui veut que l'accès à la matière enseignée soit rendu plus facile pour l'apprenant.

vi. Pour que l'élève acquière la langue seconde avec la plus grande facilité et la plus grande exactitude, la méthode sera organisée de telle façon qu'il passe du connu à l'inconnu par petites étapes, chaque étape préparant la suivante (SSTL: 81 et PLS: 67). Le passage d'une étape à la suivante doit correspondre à une capacité accrue d'assimilation, et d'emploi des éléments de la langue. Cette gradation s'organisera ainsi : la forme orale précédera la forme écrite ; l'entraînement à la réception se fera avant la lecture ; l'étudiant reproduira d'abord ce qu'il a immédiatement entendu avant de pratiquer la répétition différée; le travail en groupe précédera le travail individuel et le travail dirigé arrivera avant le travail libre (PLS., ch. 10). Les éléments linguistiques retenus pour l'enseignement seront organisés principalement selon le principe de concrétude (concreteness), fondé sur la fréquence d'occurrence des unités, sur leurs compatibilités etc. (SSTL : 86, 165 et PLS : 81 et suivantes).

\section{Synthèse}

Palmer est sans doute l'un des premiers méthodologues à esquisser un programme complet d'enseignement qui tienne compte de l'apprenant et de ses capacités d'apprentissage (PLS : 101) tout autant que de l'organisation de la langue à enseigner ; il ne néglige pas l'activité enseignante non plus (SSTL : 238-267). Palmer systématise et élargit des thèmes et des réflexions déjà présents chez Sweet et Jespersen. Sa démarche 
méthodologique tient compte des motivations et des objectifs des étudiants et propose un programme d'enseignement linguistique progressif et cohérent.

\section{Les exercices dans la pédagogie des langues de Palmer}

La première étape s'étale sur au moins un trimestre scolaire et comprend une trentaine d'heures de phonétique et d'assimilation auditive libre (LLB : 108). Ce qui est privilégié, ce sont d'abord des exercices de compréhension, sollicitant peu d'efforts (subconscious comprehension) et des exercices systématiques (imperative drills). Durant cette période, l'apprenant est également soumis à des exercices intensifs de discrimination auditive, de prononciation et d'audition, et à des exposés en langue étrangère. Les phrases utilisées lors des exercices de prononciation sont choisies parmi celles qui serviront, par la suite, pour les exercices de substitution, lors de la seconde phase d'enseignement. Durant ce bain linguistique initial, l'étudiant n'est pas censé s'exprimer en langue étrangère; il a recours à la notation phonétique pour lire et écrire.

La seconde étape vise la maîtrise de l'expression et de la compréhension orale et l'initiation à l'écrit. Cette phase s'étend sur une période qui va d'une à trois années scolaires. L'exercice de base est l'exercice de question-réponse. Divers paliers sont ménagés pour développer l'expression orale dirigée, et pour l'initiation à la lecture et à l'écriture en langue cible. Il est également question d'exercices systématiques de substitution (systematic substitution exercises) et d'exercices ergoniques systématiques (systematic ergonic exercises); ces exercices de combinatoire exploitent les possibilités de choix paradigmatiques, un peu comme les exercices à trous. L'objectif général est de faire acquérir par mémorisation la primary matter - le noyau de la langue - afin de conduire l'apprenant vers des formes de conversation libre. 

comprendre l'essentiel de ce qu'il entend et de ce qu'il lit et (peut) employer simplement et de façon relativement correcte, oralement et à l'écrit, $75 \%$ de ce qui est attesté dans le discours usuel d'un locuteur (natif) moyen» (SSTL: 118). Il s'agit dorénavant de faire acquérir les mécanismes majeurs de la langue, non acquis jusquelà. Le livre de lecture dont le vocabulaire a été sélectionné avec soin (LLB : 117) est l'instrument de base de cette étape. Plusieurs paliers consacrés à des exercices de conversation, de rédaction dirigée, de rédaction libre et de traduction sont aménagés au sein de la troisième étape.

\section{Les exercices}

Dans son souci de pédagogie concrète, Palmer distingue avec soin les exercices de production et les exercices de compréhension. Il sépare les exercices qui favorisent l'assimilation non consciente (unconscious) ou implicite, de ceux consacrés à l'assimilation consciente (conscious) ou explicite. Palmer différencie également la conversation libre, de la conversation conventionnelle, qui est une conversation aménagée et la rédaction libre de la rédaction aménagée. Il distingue les drills, qui requièrent une réponse physique et les exercices de production, de compréhension et les conversations dirigées. Il faut ajouter cependant que tout exercice peut faire l'objet de drills, c'est-à-dire d'exercices systématiques et intensifs.

31 Dans SSTL, Palmer propose quarante-sept types d'exercices dont il fournit des exemples; ces exercices sont susceptibles d'être employés aux trois étapes du cursus d'enseignement qu'il dessine (SSTL : 138 et suivantes). L'essentiel dans OM est consacré à une minutieuse typologie d'exercices pour l'oral; au moins cinquante types d'exercices sont recensés. Dans le Memorandum (1924: 23 et suivantes), Palmer identifie sept groupes d'exercices spécifiques pour l'enseignement du matériau premier (primary matter) en langue étrangère :

1. des exercices d'entraînement auditif (Ear-training Exercises),

2. des exercices d'entraînement articulatoire (Articulation Exercises),

3. des exercices de répétition (Repetition Exercises),

4. des exercices de reproduction (Reproduction Exercises),

5. des exercices de substitution (Substitution Exercises),

6. des exercices systématiques (Imperative Drill),

7. des conversations aménagées (Conventional Conversation).

Dans le Memorandum (37 et suivantes), il propose également une batterie d'exercices graphiques et oralo-graphiques, dont l'auto-dictée par exemple, pour l'acquisition du matériau écrit en langue étrangère.

Dans TOE (1940), Palmer change de perspective. Tout en ne renonçant pas à des considérations sémasiologiques, il s'oriente vers une démarche onomasiologique (Wilkins 1976). Cette orientation sera reprise dans l'élaboration du Cadre européen commun de référence pour l'enseignement des langues (CECRL) à travers l'inventaire des fonctions et des notions. Les chapitres de TOE portent tous un titre du type How to teach (Comment enseigner?) suivi d'une notion, par exemple (place and position (espace et localisation), ou time (temps), ou possession (appartenance) etc. À partir des entrées

Documents pour l'histoire du français langue étrangère ou seconde, 62-63 | 2019 
notionnelles, Palmer propose des batteries d'exercices et de procédés pour faire acquérir la notion, objet du chapitre.

\section{Synthèse}

Les types d'exercices identifiés par Palmer ont été repris dans le cadre des méthodologies audio-orales mises en place aux États-Unis, dans les années cinquante; l'exercice structural (pattern drill ou pattern practice) est devenu l'activité pédagogique type (Besse 1975). Les propositions d'exercice formulées par Palmer ont très largement alimenté les procédés d'enseignement et les méthodes en usage en didactique des langues.

L'évolution de Palmer d'une insistance sur la combinatoire ergonique, comme dans SSTL ou le Memorandum vers une approche notionnelle-fonctionnelle des constructions à enseigner, comme dans TOE, mérite d'être soulignée.

\section{Palmer et la Didactique des langues étrangères}

Tout en s'inscrivant dans le droit fil des travaux de Sweet et de Jespersen, Palmer a parachevé les travaux de ses devanciers sur la gradation et la progression du contenu linguistique à enseigner. Il a également conduit une réflexion sur les situations d'enseignement et sur les motivations des apprenants. Palmer a traduit dans les cours et manuels qu'il a rédigés, la doctrine de la nécessaire coercition des apprenants pour un meilleur résultat de l'apprentissage linguistique. À la suite de Smith (2004:246), on peut poser que l'inventivité pédagogique de Palmer constitue l'un de ses apports les plus remarquables à une pédagogie scientifique des langues. En effet, des procédés pédagogiques comme les tables de substitution, les descriptions ergoniques, les drills, les listes de constructions classées selon les notions qu'elles véhiculent, font dorénavant partie des acquis en didactique des langues.

Adossée à une psychologie associationiste, la pratique d'enseignant et de méthodologue de Palmer se fonde sur une démarche originale en lexicologie et en grammaire, démarche qui a évolué entre 1917 et 1940. Dès les années 20, il répond à l'essentiel des questions qui seront ultérieurement abordées par l'applied linguistics américaine (à partir des années quarante), puis par la didactique des langues étrangères d'expression française, à partir des années soixante-dix (Véronique 2009).

L'œuvre de Palmer porte la trace d'un fort investissement dans l'enseignement du contenu linguistique d'un point de vue sémasiologique. Pourtant en 1940, TOE montre une inflexion de sa réflexion pédagogique. Dans cet ouvrage, il aborde le contenu à enseigner en fonction des objectifs sémantiques et pragmatiques à atteindre. Cette évolution de la démarche de Palmer le rapproche de celle de Charles Bally (1865-1947), linguiste saussurien et enseignant de langues étrangères; ce que lui signale d'ailleurs Sechehaye qui établit, lui-même, ce parallèle dès 1933. Ainsi, les réflexions de Palmer sur les exercices de langue révèlent un enseignant qui allie habilement des réflexions linguistiques et pédagogiques novatrices, pour les asservir aux tâches pratiques d'enseignement et d'apprentissage linguistique. Palmer illustre ainsi sans doute une des premières figures modernes du didacticien des langues étrangères. 


\section{Sources primaires}

PALMER, Harold. E. (1968 [1917]). The scientific study and teaching of languages. London : Oxford University Press. En ligne : [https://archive.org/details/in.ernet.dli.2015.90560/page/n177]. PALMER, Harold. E. (1965 [1921]). The oral method of teaching languages. Cambridge: Heffer. PALMER, Harold. E. (1964 [1922]. The principles of language study. London : Oxford University Press. PALMER, Harold. E. (1924). Memorandum on problems of English teaching in the light of a new theory. Tokyo : Institute in Research for English Teaching. PALMER, Harold. E. \& REDMAN, Vere (1969 [1932]). This language learning business. Oxford : Oxford University Press. En ligne : [https://files.eric.ed.gov/fulltext/ED044962.pdf]. PALMER, Harold. E (1958 [1940]). The teaching of oral English. London : Longmans, Green and Co.

\section{Sources secondaires}

BALLY, Charles (1921). Traité de stylistique française. Heidelberg : Carl Winter's

Universitätsbuchhandlung.

BALLY, Charles (1925). Le Langage et la vie. Genève : Librairie Droz.

BESSE, Henri (1975). « De la pratique aux théories des exercices structuraux ». Études de linguistique appliquée 20, 8-30.

FRIES, Charles (1952). The structure of American English. New York : Harcourt, Brace \& World.

GALAZZI, Enrica (2000). «L'association phonétique internationale ». In Sylvain Auroux (dir.).

Histoire des idées linguistiques. Tome 3. Sprimont : Mardaga, 499-516.

PUREN, Christian (1988). Histoire des méthodologies de l'enseignement des langues. Paris : Clé International.

SECHEHAYE, Albert (1908). Programme et méthodes de la linguistique théorique - Psychologie du langage. Paris : Honoré Champion.

SECHEHAYE, Albert (1934). « A personal tribute to Mr. H.E. Palmer ». In Naoe Naganuma (ed.), A Commemorative Volume Issued by the Institute for Research in English Teaching on the Occasion of the Tenth Annual Conference of English Teachers Held under its Auspices. Tokyo : Kaitakusha, 10-14. En ligne : [https://warwick.ac.uk/fac/soc/al/research/collections/elt_archive/ halloffame/palmer/archive/sechehaye_1934.pdf].

SMITH, Richard C. (1999). The writings of Harold E. Palmer. An overview. Hon-no-Tomasha : Tokyo. SMITH, Richard C. (2004). An investigation into the roots of ELT, with a particular focus on the career and legacy of Harold E. Palmer (1877-1949). PhD, U. of Edinburgh. En ligne : [https:// www.era.lib.ed.ac.uk/handle/1842/6750]. 
SMITH, Richard C. (2011). « Harold E. Palmer's alternative 'applied linguistics'« . Histoire Épistémologie Langage. Linguistique appliquée et disciplinarisation, tome 33, fascicule 1, 53-67. En ligne : [https://www.persee.fr/doc/hel_0750-8069_2011_num_33_1_3206].

VÉRONIQUE, Georges Daniel (1983). Analyse contrastive, analyse d'erreurs. Une application de la linguistique à la didactique des langues II. Thèse, U. de Provence.

VÉRONIQUE, Georges Daniel (1992). «Sweet et Palmer : des précurseurs de la linguistique appliquée à la didactique des langues ? Cahiers Ferdinand de Saussure 46, 173-190.

VÉRONIQUE, Georges Daniel (2009). « La linguistique appliquée et la didactique des langues et des cultures : une polémique française au cœur d'un débat international ». Le français dans le monde. Recherches et applications 46, 42-52.

VIGNER, Gérard (1984). L'exercice dans la classe de français. Paris : Hachette.

WILKINS, David A. (1976). Notional syllabuses : A taxonomy and its relevance to foreign language curriculum development. Oxford: Oxford University Press.

\section{ANNEXES}

\section{Extraits de The Scientific Study and Teaching of languages (168-180)}

Ces quelques citations de Palmer permettent de saisir la mise en œuvre du recours à l'interrogation que ce pédagogue et didacticien préconise à tous les niveaux de l'enseignement. Cette démarche « interrogative » est à la base de The teaching of Oral English, (1940) qui comporte de nombreux courts chapitres qui commencent tous par « How to teach [...] » (comment enseigner) suivi par un terme métalinguistique « the name of objects » (le nom des objets), « describing of objects and persons » (la description des objets et des personnes), «time» (le temps), etc.

Ces extraits concernent le recours à la méthode interrogative au second niveau d'enseignement, ici du français langue étrangère.

(168) : « The second or intermediate stage is the beginning of what most people would term the study proper of the language. »

(172-173) : « Systematic Questionnaire

A second line of study may be carried out concurrently conceived on the following lines :

$[\ldots]$

T. Le plafond est-il blanc?

P. Oui, il est blanc.

T. Le plafond est-il noir?

P. Non, il n'est pas noir.

T. Quelle est la couleur du plafond? 
P. II est blanc.

T. Qu'est-ce qui est blanc?

P. C'est le plafond.

[...]. »

(174) : « There is no limit to the number of model sentences which can be developed into systematic questions and answers. Any of those from the conjugation exercises will serve this purpose, as will also any incidental sentences whatever. In this type of exercise catenizing and semanticizing are performed simultaneously.

The questions may be classified and asked in the cross order :

\begin{tabular}{|l|l|}
\hline Teacher & Pupil \\
\hline Qu'est-ce que c'est? & C'est la fenêtre. \\
\hline Qu'est-ce que c'est? & C'est la porte. \\
\hline Qu'est-ce que c'est? & C'est le plafond. \\
\hline Etc. & Etc. \\
\hline
\end{tabular}

[...].»

(175) : " Another form of systematic questionnaire will be based on a series of questionand-answer groups, of which the following are typical specimens :

\begin{tabular}{|l|l|}
\hline Qu'est-ce que le cheval? & C'est un animal. \\
\hline Qu'est-ce que le chien? & C'est un animal. \\
\hline Qu'est-ce que le chat? & C'est un animal. \\
\hline Qu'est-ce que le mouton? & C'est un animal. \\
\hline Qu'est-ce que la vache? & C'est un animal. \\
\hline Etc. & Etc. \\
\hline Qu'est-ce que la table? & C'est un meuble. \\
\hline Qu'est-ce que la chaise? & C'est un meuble. \\
\hline Qu'est-ce que le lit? & C'est un meuble. \\
\hline Etc. & Etc. \\
\hline
\end{tabular}

[...]. »

(179-180) : « Non-systematic Questionnaire 
After a certain amount of systematic questioning has been given the teacher may venture on questions chosen at random. If a large number of mistakes occur this work should be dropped immediately and be postponed to a more propitious moment.

\begin{tabular}{|l|l|}
\hline Qu'est-ce que c'est? & C'est le plafond. \\
\hline Est-il haut ou bas? & II est haut. \\
\hline Quelle est la couleur du plafond? & II est blanc. \\
\hline Pouvez-vous le toucher? & Non, je ne peux pas le toucher. \\
\hline Pourquoi pas? & Parce qu'il est trop haut. \\
\hline Qu'est-ce que c'est? & C'est la porte. \\
\hline Est-elle ouverte ou fermée? & Elle est fermée. \\
\hline Pouvez-vous I' ouvrir? & Oui, je peux I'ouvrir. \\
\hline Pouvez- vous toucher la porte si vous \\
restez la? & $\begin{array}{l}\text { Non, monsieur, je ne peux pas toucher la porte si je } \\
\text { reste ici. }\end{array}$ \\
\hline Pourquoi pas? & Parce que la porte est trop loin. \\
\hline
\end{tabular}

[...]. »

\section{NOTES}

1. A la suite de Christian Puren (1988: 16-18), j'emploierai «méthodologie » pour désigner des formations historiques en didactique des langues définies par leurs objectifs, leurs contenus linguistiques et culturels, leurs théories de référence, les situations d'enseignement où elles sont sollicitées et les « méthodes » qu'elles emploient. Dans ce contexte, le terme « méthode » désigne, donc, « un ensemble de procédés et de techniques de classe». Cependant, dans mon texte, le terme de "méthode» aura quelques fois le sens de méthodologie, comme dans "méthode directe » ou « méthode Palmer ».

2. De nombreux documents et renseignements sont déposés dans le Harold E. Palmer Archive de l'université de Warwick. $C f$ en ligne :

[https://warwick.ac.uk/fac/soc/al/research/collections/elt_archive/halloffame/palmer/ archive/]. Dorothée Anderson, fille de Palmer a rédigé une biographie et une bibliographie de son père dans Palmer \& Redman ([1932] 1969 : 133-166).

3. Fondée en 1888, l'Association Phonétique Internationale (API) a pris ce nom en 1897, sous l'impulsion de Jespersen (Galazzi 2000 : 502).

4. « un savant accompli dans l'étude du français et un excellent enseignant de langue » (Ma traduction).

5. « un talent remarquable en matière de théorie linguistique et de pédagogie » (Ma traduction). 


\section{RÉSUMÉS}

Cette contribution est consacrée à une étude des types d'exercices proposés par Harold E. Palmer (1877-1949) au long de ces années d'exercice comme enseignant de langues vivantes au RoyaumeUni et à l'étranger, et comme théoricien de l'enseignement-apprentissage des langues étrangères. L'article rappelle la place et le rôle de Palmer dans le champ de l'enseignement des langues en sa qualité de successeur des fondateurs de la méthodologie directe. Au long de sa carrière, Palmer a cherché à fonder une pédagogie scientifique des langues adossée à des travaux en phonétique et en linguistique, et marquée par une psychologie associationniste. Dans le sillage de la méthodologie directe, Palmer a prôné une démarche d'enseignement fondée sur une sélection du contenu linguistique à enseigner et sur une progression rigoureuse. La créativité dont il a su faire preuve en matière d'élaboration d'exercices de langues s'explique par la cohérence et la rigueur de sa pédagogie scientifique des langues étrangères.

This contribution is devoted to a study of the types of exercises proposed by Harold E. Palmer (1877-1949) during his years of practice as a modern language teacher in the United Kingdom and abroad, and as a theorist of the teaching and learning of foreign languages. The article recalls Palmer's place and role in the field of language teaching as a successor to the founders of the direct methodology. Throughout his career, Palmer has attempted to develop a scientific pedagogy of languages, based on work in phonetics and linguistics, and marked by associationist psychology. In the wake of the direct methodology, Palmer advocated a teaching approach based on the selection of the linguistic content to be taught and on a rigorous progression. The creativity he has shown in the development of language exercises may be explained by the consistency and rigor of his scientific pedagogy of foreign languages.

\section{INDEX}

Mots-clés : méthode directe, méthode orale, exercice, pédagogie scientifique, sélection, progression

Keywords : direct method, oral method, exercise, scientific pedagogy, selection, progression

\section{AUTEUR}

\section{GEORGES DANIEL VÉRONIQUE}

Aix-Marseille Université georges.veronique@orange.fr 